\title{
New Method Simulating Flexible object-operator Cube Model
}

\author{
Yu-Cai Ma ${ }^{\mathrm{a},{ }^{*}}$, Hai-Ru Long ${ }^{\mathrm{a}}$ \\ ${ }^{a}$ Textile College of Donghua University, Shanghai, China
}

\begin{abstract}
In order to represent complex graph drawing in the course of 3-dimensional dynamic simulating flexible objects such as fabric、garment、rope、netting yarn and rubber band, we designed a new mathematic model named operator cube. Any graph formed on the basis of the point information. In a word, if each point has enough geometry information, the graph can be drawn and rendered. Operator cube model came into being just because of that idea. The paper mainly discussed the theory of operator cube model. The model can transform a complex graph into a simple representation effectively and in real time, especially in the course of topology structure varying flexible object simulation. What's more, the model can accurately express the simulation results. The model converted between the world coordinate system space and device coordinate system space. And the model and the conversion involved some profound mathematic theorems and our conclusions which were proved strictly.
\end{abstract}

Index Terms: 3-dimension simulation; transformation; operator; operator cube; operator approximation

(C) 2011 Published by MECS Publisher. Selection and/or peer review under responsibility of the Research Association of Modern Education and Computer Science.

\section{Introduction}

Such as fabric and garment flexible materials simulation of pattern of deformation had attracted many researchers in the area of computer graphics from the middle to late eighties as computer technologies progressed, so all kinds of modelling technologies came into being[1]. The modelling method can be mainly classified into four categories which were the geometrical and the mechanical approach, their mixed approach and other methods [2-6]. Simulation of draping and buckling of a fabric which was mainly woven has received much attention in recent years in literature; the results have paved the way for development of a computer software which can model how the clothes made of fabric appear on, for instance, cloth draping on mannequins[7-11]. And there were also some simulation about large deformations occurring at any place in the cloth, such as folds and wrinkles [12-13]. However, these methods had given up to compromises among real-time performance、 accuracy, robustness and stability. Little work of simulations of flexible objects like

* Corresponding author.

E-mail address: ma-101@163.com 
fabric and so on has been concerned with negotiation of real-time performance、 accuracy, robustness and stability in literature[14]. It is a more difficult task to do that. To solve that problem, we first constructed a mathematic model named operator cube to give the problem theoretical solution and experimental results.

\section{Preliminaries}

Definition 1. An n-tuple of numbers $\left(\mathrm{x}_{1}, \mathrm{x}_{2}, \ldots, \mathrm{x}_{\mathrm{n}}\right)$ is called an $\mathrm{n}$-dimensional vector, individual numbers $\mathrm{x}_{1}, \mathrm{x}_{2}, \ldots, \mathrm{x}_{\mathrm{n}}$ are called the components of the vector. Vectors in geometry can be viewed as particular cases of $n=2,3$. When $n>3$ the $n$-dimensional vectors no longer have geometric senses. In the paper we take $n=3$.

Definition 2. The set of all real and $n$-dimensional vectors is denoted by $R^{n}$. We call $R^{n}$ an $n$-dimensional vector space or $n$-dimensional real linear space. That is to say, these vectors can be added and multiplied by numbers, and the following properties are satisfied:

(a) $\alpha+\beta=\beta+\alpha$;

(b) $\alpha+(\beta+\gamma)=(\alpha+\beta)+\gamma$;

(c) $\alpha+0=\alpha$;

(d) Let ${ }_{\alpha=\left(\alpha_{1}, \cdots, \alpha_{\mathrm{n}}\right)}$ and let $-\alpha=\left(-\alpha_{1}, \cdots,-\alpha_{\mathrm{n}}\right)$. Then ${ }_{\alpha+(-\alpha)=0}$;

(e) $1 \alpha=\alpha$;

(f) $k(\alpha+\beta)=k \alpha+k \beta$;

(g) $\left(k_{1}+k_{2}\right) \alpha=k_{1} \alpha+k_{2} \alpha$;

(h) $\left(k_{1} k_{2}\right) \alpha=k_{1}\left(k_{2} \alpha\right)$.

Where $\alpha, \beta, \gamma$ are $\mathrm{n}$-dimensional vectors, $\mathrm{k}_{1}, \mathrm{k}_{2}$, are numbers.

Definition 3. Consider vectors $\beta, \alpha_{1}, \alpha_{2}, \cdots, \alpha_{m}$, If there exist $\mathrm{m}$ numbers $\lambda_{1}, \lambda_{2}, \cdots \lambda_{m}$, such that $\beta=\lambda_{1} \alpha_{1}+\lambda_{2} \alpha_{2}+\cdots+\lambda_{m} \alpha_{m}$, then the vector $\beta$ is called a linear combination of the vectors $\alpha_{1}, \alpha_{2}, \cdots, \alpha_{m}$. We say also the vector ${ }_{\beta}$ can be linearly expressed by the vectors $\alpha_{1}, \alpha_{2}, \cdots, \alpha_{m}$.

Definition 4. Let $\alpha_{1}, \alpha_{2}, \cdots, \alpha_{m}$ be $\mathrm{m}$ vectors. We say that they are linearly dependent if there exist $\mathrm{m}$ numbers $\mathrm{k}_{1}, \mathrm{k}_{2} \ldots \mathrm{k}_{\mathrm{m}}$ not all equal to 0 such that ${ }_{k_{1} \alpha_{1}+k_{2} \alpha_{2}+\cdots+k_{m} \alpha_{m}=0}$. If such numbers do not exist, then we say that $\alpha_{1}, \alpha_{2}, \cdots, \alpha_{m}$ are linearly independent.

Definition 5. Suppose V is the linear space defined on number field $\mathrm{K}$, If there exist $\mathrm{n}$ linear independent vectors $\alpha_{1}, \alpha_{2}, \cdots, \alpha_{m}$, such that $\beta=\lambda_{1} \alpha_{1}+\lambda_{2} \alpha_{2}+\cdots+\lambda_{m} \alpha_{m}$ for $\forall \beta \in V$, then $\left\{\alpha_{1}, \alpha_{2}, \cdots, \alpha_{m}\right\}$ is a group of base of $\mathrm{V}, \mathrm{V}$ is called $\mathrm{m}$-dimension linear space, if $m \rightarrow \infty, \mathrm{V}$ is called infinite dimensional linear space.

Definition 6. A norm on a vector space $\mathrm{X}$ is a function $\|\bullet\|: X \rightarrow R^{+}:=[0, \infty) ; x \mapsto\|x\|$ that satisfies, for all $x, y \in X$ and $\alpha \in F$,

(a) $\|x\|=0$ if and only if $x=0$;

(b) $\|\alpha x\|=|\alpha|\|x\|$;

(c) $\|x+y\| \leq\|x\|+\|y\|$

A semi norm on $\mathrm{X}$ is a function $p: X \rightarrow R^{+}$that satisfies (b) and (c) above.

Definition 7. Suppose $\Lambda$ as real number field or complex number field, $X$ and $Y$ are linear spaces defined on field $\Lambda, \mathrm{D}$ is the subspace of $\mathrm{X}, \mathrm{T}$ is a mapping from $\mathrm{D}$ to $\mathrm{Y}$, for $x \in D$, the $\mathrm{x}$ image of mapping $\mathrm{T}$ denotes as $T x$ or $T(x)$, if for $\forall x, y \in D$ and number $\alpha, \beta \in \Lambda$, there is $T(\alpha x+\beta y)=\alpha T x+\beta T y$, then $\mathrm{T}$ is a linear operator, $\mathrm{D}$ is

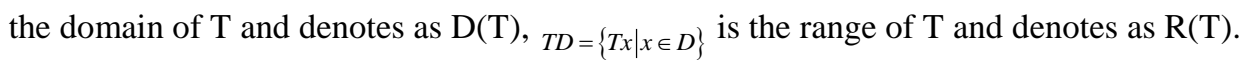




\section{Operator cube model}

\subsection{Homogeneous Coordinates}

Homogeneous coordinates are ubiquitous in computer graphics because they solve the problem of representing a transformation such as translation, projection v rotation, scaling and shear as a matrix operation. Homogeneous coordinates allow all affine transformations to be represented by a matrix operation. A translation in $\mathrm{R}^{2}:(x, y) \rightarrow(x+a, y+b)$ can be represented as

$\left[\begin{array}{lll}1 & 0 & a \\ 0 & 1 & b \\ 0 & 0 & 1\end{array}\right]\left(\begin{array}{l}x \\ y \\ 1\end{array}\right)=\left(\begin{array}{c}x+a \\ y+b \\ 1\end{array}\right)$

Where column vectors are the homogeneous coordinates of the two points.

\subsection{Common 3D transformations}

- Translation

Going from point $\mathrm{p}$ to $\mathrm{p}^{\prime}$ is by displacing by a distance $\mathrm{d} ; \mathrm{p}$ and $\mathrm{p}$ ' are points in 3-dimension vector space, here $p=(x, y, z, 1), p^{\prime}=\left(x^{\prime}, y^{\prime}, z^{\prime}, 1\right), d=\left(\alpha_{x}, \alpha_{y}, \alpha_{z}, 1\right)$ so we easily obtain the following formula $p^{\prime}=p+d \Rightarrow p^{\prime}=T p, T$ is a translation matrix, and it can be written as:

$T=\left[\begin{array}{cccc}1 & 0 & 0 & \alpha_{x} \\ 0 & 1 & 0 & \alpha_{y} \\ 0 & 0 & 1 & \alpha_{z} \\ 0 & 0 & 0 & 1\end{array}\right]$

- Scaling

Suppose $_{\beta_{x}, \beta_{y}, \beta_{z}}$ respectively as $\mathrm{x}$-direction、 $\mathrm{y}$-direction and $\mathrm{z}$-direction scaling factors relative to a

fixed point of the origin. So there is $p^{\prime}=S p$, here $S$ can be written as:

$$
S=\left[\begin{array}{cccc}
\beta_{x} & 0 & 0 & 0 \\
0 & \beta_{y} & 0 & 0 \\
0 & 0 & \beta_{z} & 0 \\
0 & 0 & 0 & 1
\end{array}\right]
$$

- Rotation

Suppose $R_{x}(\theta), R_{y}(\theta), R_{z}(\theta)$ respectively revolve $\theta$ angle around x-axis $、 \mathrm{y}$-axis and z-axis rotation matrix with a fixed point at the origin. So there is $p^{\prime}=R_{x} p$ and $p^{\prime}=R_{y} p$ and $p^{\prime}=R_{z} p$, here

$R_{x}(\theta), R_{y}(\theta), R_{z}(\theta)$ can be written as:

$$
R_{x}(\theta)=\left[\begin{array}{cccc}
1 & 0 & 0 & 0 \\
0 & \cos \theta & -\sin \theta & 0 \\
0 & \sin \theta & \cos \theta & 0 \\
0 & 0 & 0 & 1
\end{array}\right] \quad R_{y}(\theta)=\left[\begin{array}{cccc}
\cos \theta & 0 & \sin \theta & 0 \\
0 & 1 & 0 & 0 \\
-\sin \theta & 0 & \cos \theta & 0 \\
0 & 0 & 0 & 1
\end{array}\right] \quad R_{z}(\theta)=\left[\begin{array}{cccc}
\cos \theta & -\sin \theta & 0 & 0 \\
\sin \theta & \cos \theta & 0 & 0 \\
0 & 0 & 1 & 0 \\
0 & 0 & 0 & 1
\end{array}\right]
$$


- Shear
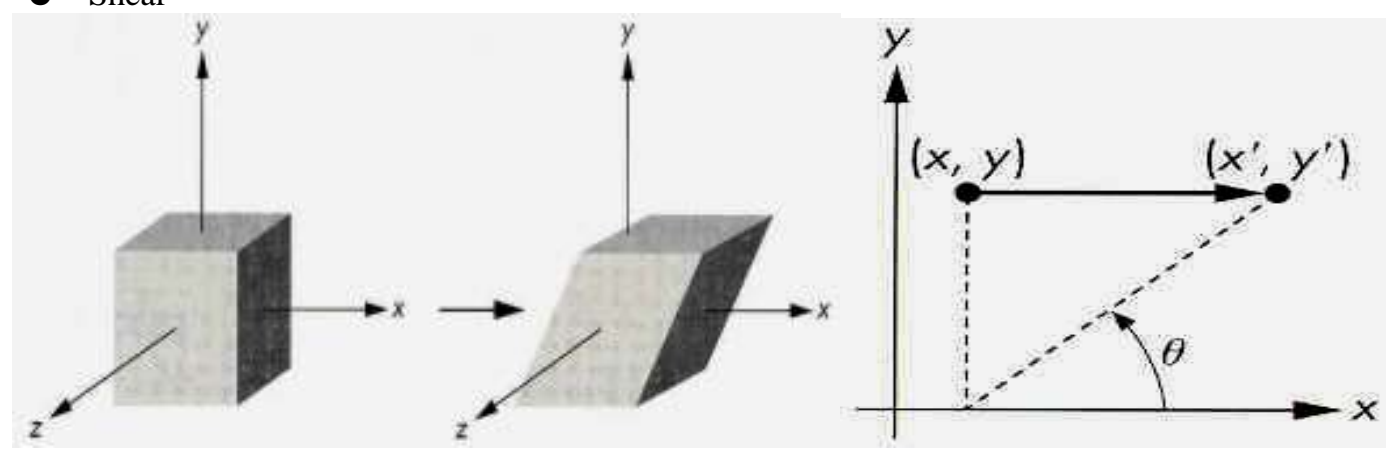

Fig. 1 Shear in the $\mathrm{x}$-axis direction

As the diagram indicated above, we can conclude

$$
\left\{\begin{array}{l}
x^{\prime}=x+y \cot \theta \\
y^{\prime}=y \\
z^{\prime}=z
\end{array}\right.
$$

So we can express it in matrix form, that is to say, $p^{\prime}=S_{x} p$, here $S_{x}$ can be written as:

$$
H_{x}=\left[\begin{array}{cccc}
1 & \cot \theta & 0 & 0 \\
0 & 1 & 0 & 0 \\
0 & 0 & 1 & 0 \\
0 & 0 & 0 & 1
\end{array}\right]
$$

If the shear happens in the y-axis or z-axis direction, we can obtain the following conclusions in the same reason.

$$
H_{y}=\left[\begin{array}{cccc}
1 & 0 & 0 & 0 \\
0 & 1 & \cot \theta & 0 \\
0 & 0 & 1 & 0 \\
0 & 0 & 0 & 1
\end{array}\right] \quad H_{z}=\left[\begin{array}{cccc}
1 & 0 & 0 & 0 \\
0 & 1 & 0 & 0 \\
\cot \theta & 0 & 1 & 0 \\
0 & 0 & 0 & 1
\end{array}\right]
$$

- Projection

Suppose $p=(x, y, z)$ is a point in the three-dimensional space, the $p^{\prime}=(x, y, 0)$ and $p^{\prime \prime}=(x, 0,0)$ is the point $p$ projection onto the xy-plane and x-axis, set $p^{\prime}=P_{x y} p$ and $p^{\prime \prime}=P_{x} p$, here $P_{x y}$ and $P_{x}$ can be written as:

$$
P_{x y}=\left[\begin{array}{cccc}
1 & 0 & 0 & 0 \\
0 & 1 & 0 & 0 \\
0 & 0 & 0 & 0 \\
0 & 0 & 0 & 1
\end{array}\right] \quad P_{x}=\left[\begin{array}{llll}
1 & 0 & 0 & 0 \\
0 & 0 & 0 & 0 \\
0 & 0 & 0 & 0 \\
0 & 0 & 0 & 1
\end{array}\right]
$$




\subsection{Operator Cube}

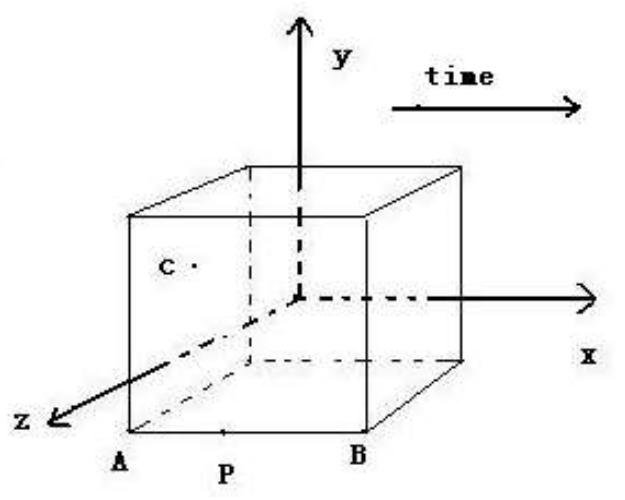

Fig. 2. Operator cube

To solve the problem of deformation in the simulation of flexible objects, we constructed a model named operator cube to represent the information of topological structure. Whether the topological structure varied or not, we use the model to convert the change of points on the object into operator on or inside the operator cube and we obtained sequence of operators. Then we added time perturbation to sequence of operator system and we inversely transformed the sequence of operator system to the simulation result. We can control the process of simulation to avoid the unexpected such as super-elasticity and misconvergence by using the model.

Operator cube is 4-dimensional cube. And it is composed of time operator and a solid cube whose elements are consisted of operators. Operators on the surface and inside the operator cube have different properties. What's more, the edge and vertex of the operator cube are made of different operators. The idea of operator cube model arose from three-dimensional orthogonality of flexible objects. The following is about the theory of operator cube.

- Operators on the faces

Each face of the operator cube is regarded as normalized operator space X. Each matrix is regarded as an operator, so the six faces are on the base of translation operator T、 scaling operator SG、rotation operator R、 shear operator $\mathrm{S}$ and projection operator P. Because these five operators are linearly independent, they are a group of base of $\mathrm{X}$. thus each operator on the face can be linearly expressed by the group of base. Each operator $\mathrm{O}$ on the face is written as

$$
\mathrm{O}=\left(\mathrm{x}_{1} \mathrm{~T}+\mathrm{x}_{2} \mathrm{SG}+\mathrm{x}_{3} \mathrm{R}+\mathrm{x}_{4} \mathrm{~S}+\mathrm{x}_{5} \mathrm{P}\right) \Delta \mathrm{T}
$$

Here $\mathrm{X}=\left(\mathrm{x}_{1}, \mathrm{x}_{2}, \mathrm{x}_{3}, \mathrm{x}_{4}, \mathrm{x}_{5}\right)$ is the operator coefficient and is the time of physical parameters and mesh spatial relationship[15-16]. A flexible object may have many physical parameters, we made use of grey system and neural network to gain optimal parameters and mesh spatial relationship depended on what kind of flexible objects were simulated. $\Delta \mathrm{T}$ is a time operator which controlled time step. when $\Delta \mathrm{T}$ is unit operator, the modelling is based on geometrical approach.

The cube has six faces which locate in the left、 in the right、 in the front、 in the back、 on top、on bottom. These faces correspond to real flexible cubic object's faces, so each of $T 、 S G 、 R 、 S 、 P$ needed to be expressed as six operators which were $T_{\text {left }} 、 T_{\text {right }} 、 T_{\text {front }} 、 T_{\text {back }} 、 T_{\text {top }} 、 T_{\text {bottom }}, S_{\text {left }}$, $S G_{\text {right }} 、 S_{\text {front }} 、 S_{\text {back }} 、 S G_{\text {top }} 、 S_{\text {bottom }} 、 R_{\text {left }} 、 R_{\text {right }} 、 R_{\text {front }} 、 R_{\text {back }} 、 R_{\text {top }} 、 R_{\text {bottom }} 、 S_{\text {left }} 、 S_{\text {right }} 、$ $\mathrm{S}_{\text {front }} 、 \mathrm{~S}_{\text {back }}, \mathrm{S}_{\text {top }}, \mathrm{S}_{\text {bottom }} 、 \mathrm{P}_{\text {left }}, \mathrm{P}_{\text {right }}, \mathrm{P}_{\text {front }} 、 \mathrm{P}_{\text {back }} 、 \mathrm{P}_{\text {top }} 、 \mathrm{P}_{\text {bottom }}$. 
- Operators on the edges

Suppose $\mathrm{O}_{1}$ and $\mathrm{O}_{2}$ are operators on the different faces of the cube, because real flexible objects are isotropic materials, the cube was symmetric. And an edge is the intersection of the two different faces, and then the operator $\mathrm{O}$ on an edge was calculated by

$\mathrm{O}=\left(\mathrm{O}_{1}+\mathrm{O}_{2}\right) / 2$

- Operators on the vertices

Suppose $\mathrm{O}_{1} 、 \mathrm{O}_{2}$ and $\mathrm{O}_{3}$ are operators on the different edges of the cube; because real flexible objects are isotropic materials, the cube was symmetric. And a vertex is the intersection of the three different edges, and then the operator $\mathrm{O}$ on an edge was calculated by

$\mathrm{O}=\left(\mathrm{O}_{1}+\mathrm{O}_{2}+\mathrm{O}_{3}\right) / 3$

- Operators inside the cube

Because our operator cube was regarded as micro-unit cube on the Euclidean Space, each operator inside the cube was similar to a point inside the unit cube on the Euclidean Space. We could make an operator inside the cube project to three different edges sharing the same vertex, thus we could get an operator's orthogonal decomposition. Considered an $\mathrm{O}$ operator inside the cube, and suppose $\mathrm{O}_{1}$ 、 $\mathrm{O}_{2} 、 \mathrm{O}_{3}$ were $\mathrm{O}$ operator's projections on the edges sharing the same vertex, then the operator $\mathrm{O}$ could be written as

$\mathrm{O}=\mathrm{O}_{1}+\mathrm{O}_{2}+\mathrm{O}_{3}$

- Time operator

Different time step has great influence on simulation when taken in a mechanical approach, so it was a big problem to take optimal time step. We used to have different tests to find suitable time step, and we adopted a self-adaptive way to control simulation through time operator. Thus we could use absolute error to control simulation. Time operator was related to numerical method, and different numerical methods were endowed with different time operators.

\section{Example and experiment}

When the flexible objects deformed in the course of simulation, if the object topological structure didn't change, we can represent deformation by deformation gradient. And deformation gradient is generally matrix of a linear transformation, what' more, it contains geometric information in the process of deformation. It is obvious that we can decompose deformation gradient into operators inside or on the operator cube; in other words, operator cube can describe the information which the deformation gradient contains. If the object topological structure changed in the course of simulation, for example fracture, we can represent deformation by generalized deformation gradient and operator cube can describe the information which the generalized deformation gradient by using the theory of operator decomposition and operator approximation. To test our model, we translated our ideas into computer code and gained the following experiment result as shown in the picture below. 


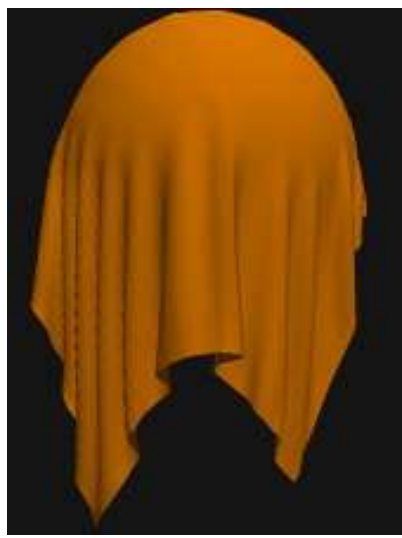

Fig. 3. Fabric drape

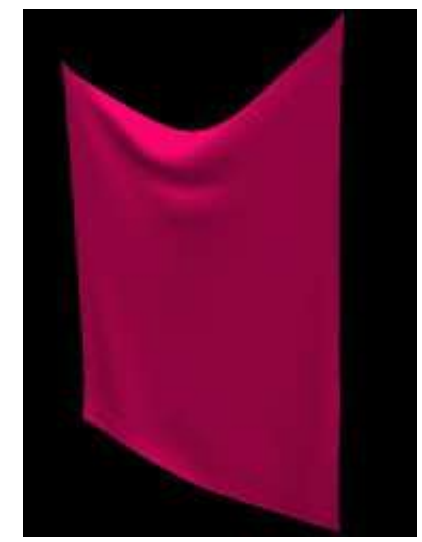

Fig. 4. Fabric suspension

\section{Conclussion and discussion}

This paper described a new mathematic model named cube operator. And this model was a good approximation of simulation method. The model successfully represented uniform theory of geometrical and mechanical approach and also mixed approach. Actually we could gain expected simulation results through this model only if we could find appropriate operator sequence and physical parameters. But our model is not perfect, because this model could only characterize the isotropic material simulation. How to characterize the anisotropic material and multilayer structure simulation is a big problem, and how to blend texture and other information into cube operator model also is a problem, we will aim to solve these work in the future.

\section{Acknowledgements}

The author wished to thank Professor Hai-Ru Long for suggesting this problem and for discussing various ideas relating to this work. I would also like to acknowledge the useful suggestions made by the referee. 


\section{References}

[1] [Nadia Magnenat Thalmann, Pascal Volino. From erly draping to haute couture models: 20 years of research[J].Visual Computer, 2005, 21:506-519.

[2] David E Breen, Donald H House. A Particle-Based Model for Simulating the Draping Behaviour of Woven Cloth[J].Textile Research Journal, 1994, 64(11):663-685.

[3] D M Stump, W B Fraser. A Simplified Model of Fabric Drape Based on Ring Theory[J].Textile Research Journal, 1996, 66(8):506-514.

[4] Pascal Volino, Nadia Magnenat-Thalmann. Stop-and-go cloth draping[J].Visual computer, 2007, 23:669677.

[5] Ming-xiang Chen, Qing-ping Sun, Ming-fai Yuen. Simulation of Fabric Drape Using A Thin Plate Element with Finite Rotation[J].Acta Mechanica Sinica, 1998, 14(3):239-247.

[6] Bijian Chen, Muthu Govindaraj. A Physically Based Model of Fabric Drape Using Flexible Shell Theory[J].Textile Research Journal, 1995, 65(6):324-330.

[7] L Dong, C Lekakou, M G Bader. Solid-mechanics finite element simulations of the draping of fabrics: a sensitivity analysis[J]. composites: Part A, 2000, 31:639-652.

[8] S F Chen, J L Hu, J G Teng. A finite-volume method for contact drape simulation of woven fabrics and garments[J].Finite Elements in Analysis and Design, 2001, 37:513-531.

[9] K Y Sze, X H Liu. Fabric drape simulation by solid-shell finite element method [J]. Finite Elements in Analysis and Design, 2007, 43:819-838.

[10] Jacqueline R Postle, Ron Postle. The Dynamics of Fabric Drape[J].Textile Research Journal, 1999, 69(9):623-629.

[11] W M Lo, J L Hu, L K Li. Modeling a Fabric Drape Profile[J].Textile Research Journal, 2002,72(5):454463.

[12] Feng Ji, Ru-qin Li, Yi-ping Qiu. Simulate the Dynamic Draping Behaviour of Woven and Knitted Fabrics[J].Journal of Industrial Textiles, 2006, 35(3):201-215.

[13] Jose Miguel S Dias, Manuel N Gamito. A Discretized Linear Model for Cloth Buckling and Drape[J].Textile Research Journal, 2000, 70(4):285-297.

[14] Moon Koo Kang, Jeongjin Lee. A real-time cloth draping simulation algorithm using conjugate harmonic functions[J].Computers \& Graphics, 2007, 31:271-279.

[15]Bijian Chen, Muthu Govindaraj. A Parametric Study of Fabric Drape[J].Textile Research Journal, 1996, 66(1):17-24.

[16] In Hwan Sul, Tae Jin Kang. Simulation of Cusick Drapemeter Using Particle-based Modeling:Stability Analysis of Explicit Integration Methods[J].Textile Research Journal, 2006, 76(9):712-719.

[17] Kunii T. singularity theoretical modeling and animation of garment wrinkle formation process[J].visual computer, 1990,6(6):326-336.

[18] Jonathan M K,Dong L J.simulating knitted cloth at the yarn level [J]. ACM transactions on graphics,2008,27(1):1-9.

[19] Hidefumi W,Shinichi H.static modeling of linear object deformation based on differential geometry[J].the international journal of robotics research,2004,23(3):293-311.

[20] Yitong Z,Cuiyu L.a micro-mechanical model of knitted fabric and its application to the analysis of buckling under tension in wale direction:micro-mechanical model[J].acta mechanica sinica,2004,20(6):623631.

[21] Frederic C,Nadia M T.a data-driven approach for real-time cltohes simulation[J].computer graphics forum,2005,24(2):173-183.

[22] [204]Georgii J, Westermam R. a multigrid frame work for real-time simulation of deformable bodies[J].computer \& graphics, 2006,30:408-415.

[23] Jansson J. a discrete mechanics model for deformable bodies[J].computer aided design,2002,34:913-928.

[24] Gregire M, Schomer E. interactive simulation of one-dimensional flexible parts[J].computer aided 
design,2007,39:694-707.

[25] Rony G,David H.efficient simulation of inextensible cloth[J].ACM transactions on graphics,2007,26(3):1-7.

[26] Liang M,Jinlian H.generating seams and wrinkles for virtual clothing [C].proceedings of 2006 ACM interational conference on virtual reality continuum and its applications,2006,205-211.

[27] Chou K, Sun H. deformable simulation using force propagation model with finite element optimization[J].computer \& graphics,2004, 28:559-568.

[28] Elliot E,Robert B.animating developble surfaces using nonconfoming elements[J].ACM transactions on graphics,2008,27(3):1-5. 